Ayman O. Soubani Johan Decruyenaere

\section{Improved outcome of critically ill patients with hematological malignancies: what's next?}

Received: 14 July 2014

Accepted: 18 July 2014

Published online: 1 August 2014

(C) Springer-Verlag Berlin Heidelberg and ESICM 2014

\section{A. O. Soubani (®)}

Division of Pulmonary, Critical Care and Sleep Medicine, Wayne State University School of Medicine, 3990 John R-3 Hudson, 48201 Detroit, MI, USA

e-mail: asoubani@med.wayne.edu

Tel.: +313-7458471

J. Decruyenaere

Intensive Care Department, Ghent University Hospital, Ghent, Belgium

The overall care and outcome of patients with cancer, especially hematological malignancies (HM), have improved significantly over the years [1]. Aggressive and effective chemotherapeutic and surgical interventions associated with longer term survival have led to an increase in the number of patients requiring intensive care unit (ICU) care. For many years, it was assumed that the outcome of critically ill patients who required ICU care, especially mechanical ventilation, was poor and that many of those patients should not be admitted to the ICU. However, several studies since the turn of the century have shown that the outcome of critically ill patients with HM has been improving [2] (Fig. 1). In an article recently published in Intensive Care Medicine, van Vliet and colleagues explored the trends in admissions and outcomes of critically ill patients with HM and compared them to those of other equally critically ill patients [3]. In addition to providing further evidence that the outcome of these patients is improving, the authors report several intriguing observations that warrant further discussion.
Their study shows that the rate of ICU admission for patients with HM has been steadily increasing at an average annual rate of $6 \%$, which is similar to those of patients with solid malignancies. This increase coincides with an annual improvement in outcome over the study period-an average of $7 \%$ per year. Importantly, the rate of improvement was comparable to that of the overall ICU population and other equally controversial groups of patients, such as those with solid tumors, chronic liver cirrhosis and chronic heart failure. The study shows that the risk adjusted mortality in critically ill patients with HM was similar to those with solid tumors, which is a better outcome than that reported in previous studies suggesting that patients with HM had worse outcome [4]. The accelerated improvement in outcome of critically ill cancer patients in the last two decades compared to historical data probably reflects both the availability of more effective and targeted therapeutic drugs with less toxicity and better diagnosis and management of opportunistic infections that are unique to these patients. Other important factors that likely influenced the better outcome include the improvement in supportive measures for hematological disturbances in patients with HM, such as neutropenia and thrombocytopenia, prevention of tumor lysis syndrome and the multidisciplinary approach between hematologists, intensivists and other specialists in the care of these patients. However, now that these approaches and measures have been well established in majority of modern ICUs and a part of the culture of ICU care, the study suggests that there is no difference in the risk-adjusted mortality between the different groups of patients, including those with hematological and solid malignancies.

Another important finding in the study of van Vliet and colleagues is that leukopenia was not a predictor of worse short-term outcome and there was no difference in mortality between those with or without leukopenia (white blood cell $<1.0 \times 10^{9} / \mathrm{L}$ ). This finding is significant since 


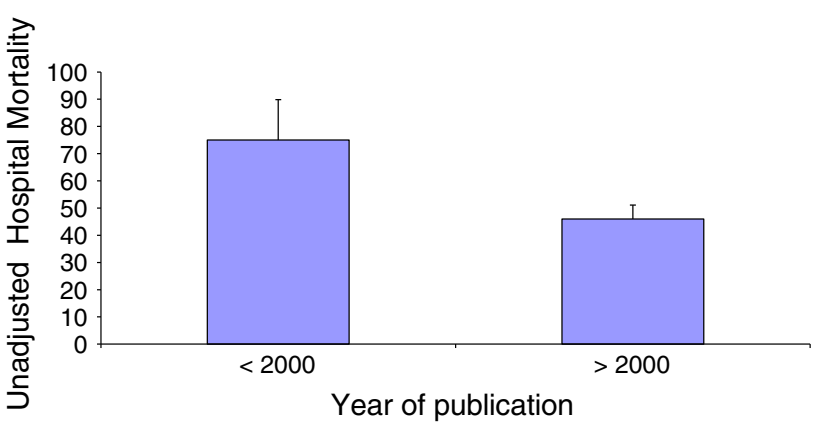

Fig. 1 Average unadjusted hospital mortality of critically ill cancer patients in studies published before and after the year 2000 (adapted from reference [18])

$23 \%$ of critically ill patients with HM in their study had leukopenia. Although this observation may not apply to severe neutropenia, it is clear that those patients had neutropenia and that their neutropenia was not associated with worse outcome. This finding corroborates the results of several other studies over the last years that showed improved outcome of critically ill patients with neutropenia [5]. Most of these studies also showed that neutropenia was not an independent predictor of poor outcomes and that similar to other groups of critically ill patients, other factors, such as the number and type of multisystem organ failure, were more important. The improvement in outcome of patients with neutropenia can be related to the improved management of severe sepsis, which is the primary reason for admission to the ICU, and judicious use of granulocyte colony-stimulating factors to diminish the risk and duration of neutropenia [6]. However, there still remain challenges in the management and outcome of neutropenia associated with complex situations, such as prolonged neutropenia, acute respiratory failure requiring mechanical ventilation, hematopoietic stem cell transplantation and noninfectious diagnoses $[6$, 7].

An important observation by van Vliet and colleagues is that the variability in volume of cancer patients between the different ICUs did not affect the outcome of these patients. Previous studies have shown that increased familiarity with the clinical spectrum and care of critically ill cancer patients was associated with better outcome [8]. It is possible that the current finding suggests that there should be more emphasis on the quality of the ICU care in its entirety, especially in areas of sepsis, mechanical ventilation, compliance with evidence-based bundles of care, nursing care and multidisciplinary collaboration in the care of critically ill patients rather than on the case volume of specific diseases, such as patients with HM.

On the other hand, the findings in the study by van Vliet and colleagues may be unique to the Netherlands. It is important to note that the data from 36 ICUs probably represent a small number of all the ICUs in the country. It is also possible that these ICUs were in tertiary or large hospitals that are more likely to care for patients with complicated HM and have more resources, multiple specialists and robust quality and safety measures in place. As such, the finding by van Vliet and colleagues that case volume did not correlate with outcome may not apply to all ICUs, and more studies are needed that correlate case volume, resources available in the ICUs and outcome of critically ill patients with HM.

The positive findings of these authors, especially in the backdrop of multiple other studies that have shown improvement in the outcome of critically ill patients with HM, add more pressure to take such gains to the next steps in the care of these patients.

We believe that more robust prospective, multicenter and multinational studies are needed which address specific issues related to the critical care of cancer patients. The study by van Vliet and colleagues represents an important step in this direction. Similar milestone studies have been published from France and Belgium and from Brazil [9, 10]. However, more studies are needed which provide more insight into the role of type and status of malignancy, the timing and reason for admission to the ICU and the nature and number of organ failure in predicting the outcome of ICU admission. More studies are also needed that are interventional rather than purely observational. Such interventions may include testing and assessing specific admission and discharge criteria, the outcome of different ventilatory strategies, including noninvasive ventilation, and diagnostic and therapeutic strategies. Few of such interventional studies have been conducted to date [11]. Future studies should also include representation from countries around the world, including developing countries, in order to improve our understanding of the attitudes, obstacles and outcomes in the care of such patients in healthcare systems that may have much fewer resources, thereby necessitating more difficult choices in patient care. The results of such studies may shed light on any gaps in the care and outcome of critically ill cancer patients and start the process of remedying such gaps.

It is clear from the study of van Vliet and colleagues that while the outcome of critically ill cancer patients is improving, these patients have higher mortality than other groups of patients and the general population of ICU patients. The authors - as did several others-recommend that ICU admission should not be withheld from critically ill cancer patients. However, many of these patients will not do well even with the best ICU care. Future studies should aim at providing guidance to clinicians when such care is futile. Previous studies provided evidence that the nature and extent of organ dysfunction 3-5 days after ICU admission are generally good predictors of outcome [12]. More prospective studies are needed to validate this observation and try to identify more specific markers of better outcome after initial aggressive ICU care. Also, 
more studies are needed on the role of palliative care and supportive care in the management of these patients. Furthermore, it is necessary to understand the psychosocial effects of critical illness on the patients and families who are dealing with the stress of critical illness and underlying cancer diagnosis. It is also important to assess the effects of critical illness on these patient's physical symptoms, such as pain and dyspnea and sleep pattern. In addition, more studies are needed which focus on the impact of providing care to critically ill cancer patients on clinicians-intensivists and nurses-especially in specialized units in cancer centers or those who care for a high volume of cancer patients. Previous studies on critical care in general have suggested there are high levels of clinician burnout and conflicts within the ICU team members and with families, especially concerning areas of goals of care [13]. These observations are likely to be accentuated in the care of patients with cancer.

Although the study by van Vliet and colleagues and other studies have shown that the short-term survival of patients with HM has improved, data on the long-term survival and consequences of critical illness in these patients are sparse. Studies on non-cancer patients have shown that critical illness is associated with long-term worsening of performance status, fatigue and cognitive impairment $[14,15]$. A recent study suggested that longterm outcome and quality of life of patients with HM admitted to the ICU are poor and that these factors should be considered in the triage process for ICU referral [16]. More studies are needed to identify the impact of critical illness and ICU care on the long-term survival of patients with cancer, including their performance status, quality of life and ability to receive further treatment for the underlying malignancy. Also, more studies will be needed to determine the impact of increasing ICU admissions of critically ill cancer patients on the healthcare cost and occupancy of ICU beds. It is evident from the data reported by van Vliet and colleagues that patients with HM had longer hospital length of stay and higher ICU readmission rate. Such data puts more emphasis on developing robust, evidence-based triage approach and admission and discharge criteria.

Another area for which there is barely any data is the biology behind the interaction between cancer and critical illness. HM and cancer treatment affect the count and function of neutrophils and $\mathrm{T}$ and $\mathrm{B}$ lymphocytes, which may in turn affect the host's defense against infection and the inflammatory responses associated with critical illness. There is evidence that septic patients with HM have impaired pro-inflammatory immune response, such as C-reactive protein, interleukin-6, neopterin, tryptophan metabolites and mannose-binding lectin levels compared to patients with non-hematological diseases [17]. Further studies are needed on the effect of cancer on the function of different organs during critical illness. Studies are also necessary to address the effects of critical illness and its treatment on cancer biology, behavior and response to therapy.

The perceived poor outcome of critically ill cancer patients that remains prevalent among members of the healthcare system, including the general population of intensivists, hematologists and oncologists, nurses, hospital administrators and health insurance providers, should be faced by disseminating the evidence-based arguments presented in the study of van Vliet and colleagues and in other similar studies. This can be achieved by more publications in general and specialized oncology, nursing and healthcare journals and presentations at meetings of professional societies on these specialties.

The findings by van Vliet and colleagues and other similar studies highlight the progress in the care of critically ill cancer patients but also underscore the fact that there is still a lot which needs to be done in this wide and exciting field.

\section{References}

1. DeSantis CE, Lin CC, Mariotto AB, et al (2014) Cancer treatment and survivorship statistics, 2014. CA: A Cancer Journal for Clinicians 64:252-271

2. Azoulay E, Soares M, Darmon M, Benoit D, Pastores S, Afessa B (2011) Intensive care of the cancer patient: recent achievements and remaining challenges. Ann Intensive Care 1:5

3. van Vliet M, Verburg IWM, van den Boogaard M et al (2014) Trends in admission prevalence, illness severity and survival of haematological patients treated in Dutch intensive care units. Intensive Care Med. doi: 10.1007/s00134-014-3373-x
4. Taccone FS, Artigas AA, Sprung CL, Moreno R, Sakr Y, Vincent JL (2009) Characteristics and outcomes of cancer patients in European ICUs. Crit Care 13:R15

5. Darmon M, Azoulay E, Alberti C et al (2002) Impact of neutropenia duration on short-term mortality in neutropenic critically ill cancer patients. Intensive Care Med 28:1775-1780

6. Legrand M, Max A, Peigne V et al (2006) Survival in neutropenic patients with severe sepsis or septic shock. Crit Care Med 40:43-49
7. Pène F, Aubron C, Azoulay E et al (2006) Outcome of critically ill allogeneic hematopoietic stem-cell transplantation recipients: a reappraisal of indications for organ failure supports. J Clin Oncol 24:643-649

8. Zuber B, Tran TC, Aegerter P et al (2012) Impact of case volume on survival of septic shock in patients with malignancies. Crit Care Med 40:55-62 
9. Azoulay E, Mokart D, Pène F et al (2013) Outcomes of critically ill patients with hematologic malignancies: prospective multicenter data from France and Belgium-a groupe de recherché respiratoire en réanimation onco-hématologique study. J Clin Oncol 31:2810-2818

10. Azevedo LC, Caruso P, Silva UV et al (2014) Outcomes for patients with cancer admitted to the ICU requiring ventilatory support: results from a prospective multicenter study. Chest. doi:10.1378/chest.13-1870

11. Azoulay E, Mokart D, Lambert J et al (2010) Diagnostic strategy for hematology and oncology patients with acute respiratory failure: randomized controlled trial. Am J Respir Crit Care Med 182:1038
12. Larché J, Azoulay E, Fieux F et al (2003) Improved survival of critically ill cancer patients with septic shock. Intensive Care Med 29:1688-1695

13. Piers RD, Azoulay E, Ricou B et al (2011) Perceptions of appropriateness of care among European and Israeli intensive care unit nurses and physicians. JAMA 306:2694-2703

14. Herridge MS, Cheung AM, Tansey CM et al (2003) One-year outcomes in survivors of the acute respiratory distress syndrome. N Engl J Med 348:683-693

15. Iwashyna TJ, Ely EW, Smith DM, Langa KM (2010) Long-term cognitive impairment and functional disability among survivors of severe sepsis. JAMA 304:1787-1794
16. Oeyen SG, Benoit DD, Annemans L et al (2013) Long-term outcomes and quality of life incritically ill patients with hematological or solid malignancies: a single center study. Intensive Care Med 39:889-898

17. Kurz K, Garimorth K, Joannidis M, Fuchs D, Petzer A, Weiss G (2012) Altered immune responses during septicaemia in patients suffering from haematological malignancies. Int $\mathbf{J}$ Immunopathol Pharmacol 1:147-156

18. Darmon M, Azoulay E (2009) Critical care management of cancer patients: cause for optimism and need for objectivity. Curr Opin Oncol 21:318-326 